\title{
Health inequalities among middle-aged and elderly people in China: analyses of cross-sectional surveys from the China Health and Retirement Longitudinal Study 2011-2016
}

Qinxiao Qiu

Soochow University

Jinfeng Zeng

Soochow University

Liyuan Han

Ningbo University

Zhuo Chen

US Centers for Disease Control and Prevention

Hongpeng Sun ( $\nabla$ hpsun@suda.edu.cn )

Soochow University https://orcid.org/0000-0001-9382-5586

\section{Research}

Keywords: Inequality, Chronic diseases, Concentration index

Posted Date: January 17th, 2020

DOI: https://doi.org/10.21203/rs.2.21234/v1

License: (c) (i) This work is licensed under a Creative Commons Attribution 4.0 International License.

Read Full License 


\section{Abstract}

Objectives: China has a history of striving to achieve health equity, including efforts to prevent and control infectious diseases. However, to date, there is no comprehensive assessment of inequalities in chronic diseases in China. Methods: Data for this study were obtained from the China Health and Retirement Longitudinal Study (CHARLS) conducted from 2011 to 2016. A total of 50,244 Chinese adults aged 45 years and older were included (16,128 in 2011, 16,646 in 2013, and 17,470 in 2015). Principal component analysis was used to construct the socioeconomic status indicator. We calculated concentration indices and corresponding Cls for 14 chronic diseases and comorbidities. We then estimated the Kendall rank correlation coefficient for inequalities and GDP per capita among provinces. Results: For 10 of the 14 chronic diseases, prevalence rates were higher for the poorest tertiles than for the richest tertiles. The concentration indices of dyslipidaemia, diabetes or high blood sugar, and cancer or malignant tumour were, respectively, 0.1256 (95\% confidence interval, 0.1052-0.151), 0.098 (0.0704$0.1244)$, and 0.1305 (0.0528-0.215) in 2015-2016, which indicated pro-rich inequality. Health inequality for chronic lung diseases and eight other diseases grew markedly from 2011 to 2016 . Overall, health inequality was lower for urban residents $(-0.035$ in $2011-2012,-0.036$ in $2013-2014$, and -0.05 in $2015-2016)$ than rural residents $(-0.053,-0.064$, and -0.08 , respectively), and inequality was twice as high among women $(-0.051,-0.05$, and -0.072 , respectively) than among men $(-0.023,-0.02$, and -0.032 , respectively). Provinces that were ranked higher for GDP per capita were also ranked higher in the degree to which disease prevalence was higher in people with lower income (Kendall's $\tau=-0.2328$, $p=0.015$; Kendall's $\tau=-0.3545, p=0.0077$; Kendall's $\tau=-0.2646, p=0.0079$, respectively). Conclusions: Propoor health inequalities for many diseases in China are large and widening. Policies associated with health equity, including free public health services and community health programmes, are needed to achieve the Sustainable Development Goals.

\section{Background}

With rapid economic development, China faces the impending challenge of an ageing society. In 2017, the number of Chinese elderly people aged 60 years or older reached 241 million, or approximately $17 \%$ of the population [1]. With an ageing population, the prevalence and attributable mortality of chronic diseases among middle-aged and elderly Chinese adults are steadily increasing because of prolonged exposure to risk factors and impairments of the immune system [2], which together have a nonlinear effect on the health of middle-aged and elderly populations. The prevalence of chronic diseases among elderly Chinese adults was estimated at $74.45 \%$ in 2010 [3].

China's health care system has made great strides in recent years, with many improved performance indicators. There was a dramatic decline in the prevalence of 30 Class A and B notifiable diseases listed in the Law of the People's Republic of China on the Prevention and Treatment of Infectious Diseases, from $20 \%$ in 1949 to $0.2 \%$ in 2015. The average life expectancy in China has risen from 67.9 years in 1981 to 76.5 years in 2016. Health inequality is another performance indicator of the health care sector, defined as differences in health that are potentially avoidable, unjust, and (or) unfair [4]. Health 
inequalities that exist across different regions and communities of China have a great impact on economic and social development. Zhong estimated that the concentration indices of self-reported poor health and the prevalence of activities of daily living were -0.0102 and -0.0247 , respectively, indicating a socioeconomic gradient among elderly Chinese residents [5]. In Shanghai, life expectancy in women was 83.54 years in 2015, whereas in poorer provinces such as Qinghai Province, it was 74.17 years-a difference of 9.37 years [6]. In addition, the health inequality index of rural residents is higher than that of urban residents in China [7]. The United Nations' Sustainable Development Goal 3 aims for a 30\% reduction in premature mortality owing to noncommunicable diseases through prevention and treatment [8]. This aim will require substantial reductions among people with a high prevalence of chronic diseases caused by differences in socioeconomic status (SES). Hence, greater attention must be paid to reducing or eliminating health inequalities in China.

A number of recent studies have demonstrated that SES is the most decisive factor affecting health [9, 10]. Additional studies report that a coherent view of reproductive, newborn, maternal, and child health inequalities has been diminishing in most countries [11-16]. However, there has been no assessment of the extent of inequalities for chronic diseases in China, which is crucial for developing national health policies to reduce mortality and morbidity. Thus, we aimed to comprehensively estimate health inequalities through cross-sectional surveys by analysing differences in the prevalence of chronic diseases among middle-aged and elderly people in China with different SES levels. We will also investigate health system factors that might be correlated with health inequalities.

\section{Methods}

\section{Data sources}

In this study, we analysed data from the China Health and Retirement Longitudinal Study (CHARLS) 2011-2012 (wave 1), 2013-2014 (wave 2), and 2015-2016 (wave 3) [17, 18]. The CHARLS is a nationally representative large-scale survey targeting the population aged 45 years and above in China. The study, launched in 2011, aims to collect a set of high-quality microdata representing families and individuals aged $\geq 45$ years to analyse population ageing in China and promote interdisciplinary research; this study covers 150 counties, 450 villages and approximately 10,000 households. Study participants are followed up every 2 years. Data collection in CHARLS has been described elsewhere [19].

We excluded observations with missing values, leaving respondents aged $\geq 45$ years: $n=16,128$ in wave 1, 16,646 in wave 2, and 17,470 in wave 3 (Table 1, Additional file 1: Fig. S1-S3).

All examinations were conducted after informed consent was obtained from participants. The Biomedical Ethics Committee of Peking University approved the survey (The approval number is IRB0000105211015), and the conduct of the study adhered to the principles of the Declaration of Helsinki.

\section{Measures}


In the CHARLS, each participant was interviewed using a standardized questionnaire and underwent a medical examination. Data included sociodemographic characteristics, biometrics, lifestyle and behavioural characteristics, cardiovascular disease risk factors, health history, and medications. Each new survey respondent was queried, "Have you been diagnosed with [chronic disease] by a doctor?" In follow-up interviews, participants were asked, "Our records from your last interview show that you have had/not had [chronic disease]; is this right?" and "Have you been diagnosed with [chronic disease] by a doctor [since your last interview in the last 2 years]?" Participants recorded yes or no responses to all questions. Those who answered affirmatively to any questions were required to provide medical or hospital records.

According to the CHARLS questionnaires, 14 chronic diseases were identified: hypertension, dyslipidaemia (i.e., elevated low-density lipoprotein, triglycerides, and total cholesterol or low high-density lipoprotein), diabetes (i.e., high blood sugar), cancer or malignant tumour (except minor skin cancers), chronic lung disease (e.g., chronic bronchitis or emphysema) excluding tumours or cancer, liver disease (except fatty liver, tumours, and cancer), heart disease (e.g., coronary heart disease, angina, congestive heart failure, other heart problems), stroke, kidney disease (except tumour or cancer), stomach and other digestive diseases (except tumour or cancer), emotional or psychiatric problems, memory-related disease, arthritis or rheumatism, and asthma.

\section{Socioeconomic status (SES)}

To measure inequalities in the prevalence of chronic disease among people with different standards of living, data on household assets and housing characteristics were used to construct a proxy index to measure living standards [20]. In this study, durable consumer goods (including an automobile, electric bicycle, motorcycle, refrigerator, washing machine, television, computer, stereo system, video camera, camera, air conditioner, mobile phone, furniture, musical instrument, valuable decorative items, jewellery, collectibles, precious metals, or artwork) and housing characteristics (including the type of structure of residence, one-story or multi-level building, toilet, electricity, running water, bathroom facilities, coal gas or natural gas, heating, source of cooking fuel, telephone, and internet connection) were combined into an index of SES to measure household living standards.

Principal component analysis (PCA) is a common approach [21] used to describe variation in a set of variables as linear combinations of the original variables, in which each continuous linear combination is derived to explain variation in the original data as much as possible while being uncorrelated with other linear combinations. Typically, the first principal component with the largest amount of information from the original variables was chosen to represent wealth status and be defined as the wealth index here [20]. In the case of PCA, the wealth index for individual is defined as follows: (see Equation 1 in the Supplemental Files)

where is the value of asset for household, is the sample mean, is the sample standard deviation, and are the weights associated with the first principal component (Additional file 1: Table S1). 


\section{Other variables}

To compare the differences in chronic disease prevalence between different participant groups, demographic characteristics (including age, sex, urban or rural residence, living area) were obtained.

\section{Concentration index}

The concentration index has become one of the standard measures in the health economics literature on equality and inequality in health and health care. The concentration index is defined as twice the area between the concentration curve and the line of perfect equality (the 45-degree line), where individuals are ranked by socioeconomic level, general income, and the cumulative ranking of each individual plotted against the cumulative share of health outcomes or health care utilization [22]. Thus, in the case of no socioeconomic-related inequality, the concentration index is zero. When the outcome of interest is ill health, the convention is that the index takes a negative value when the curve lies above the line of equality, indicating that the prevalence of chronic disease is excessively concentrated among poor populations; the index takes a positive value when the curve lies below the line of equality. The concentration index can be expressed as follows: (see Equation 2 in the Supplemental Files)

where is the indicator of the health status of individual ; is the fractional rank of individual in the living standards distribution, with for the poorest and for the wealthiest individuals; and is the mean of the health outcome variables $[22,23]$. The concentration index is bound between -1 and +1 . When the concentration index is used to compare inequality across time, place, and subpopulations, calculating the concentration index for binary outcomes is potentially problematic because the possible range of the concentration index differs according to the mean of the outcome variable [24-27]. To resolve this issue, the concentration index is divided by ( 1 - mean of the outcome variable), referred to as the adjusted concentration index herein, following previous studies $[25,26]$.

\section{Statistical analyses}

We produced an initial estimation of socioeconomic inequalities in health by comparing the prevalence rates of 14 chronic diseases across wealth index tertiles. Rates were standardized for age and sex using logistic regression, including a random effect to account for clustering at the community level.

To measure socioeconomic inequalities in chronic disease prevalence among participants of CHARLS waves 1-3, we first calculated the concentration index for the prevalence of each chronic disease in each wave. After obtaining the total concentration index, we calculated concentration indices grouped by sex and urban or rural residence to compare the differences in chronic disease prevalence inequities among different populations. We used a SAS macro and the bootstrap method to estimate the concentration index and confidence interval [28]. We plotted values of the concentration index for each province against per capita gross domestic product (GDP) in the province. We used Kendall's rank correlation coefficient (Kendall's tau) to measure the strength and direction of the association between per capita gross GDP 
and concentration indices. All data preparation and data analysis were performed in SAS version 9 (SAS Institute Inc., Cary, NC, USA).

\section{Results}

We found that among the 14 chronic diseases analysed, the prevalence of arthritis and rheumatism was the highest, with $32.5 \%$ (95\% Cl: $31.8 \%-33.2 \%)$, 35.9\% (35.2\%-36.6\%), and 32.9\% (32.2\%-33.6\%) in 2011-2012, 2013-2014, and 2015-2016, respectively (Additional file 1: Tables S2-S4). These were followed by hypertension (24.6\%, 95\% Cl: $23.9 \%-25.3 \% ; 28.2 \%, 95 \% \mathrm{Cl}: 27.6 \%-28.9 \%$; and $29.7 \%, 95 \% \mathrm{Cl}$ : $29 \%-30.3 \%$ ) and stomach or other digestive system diseases (21.8\%, 95\% Cl: $21.1 \%-22.4 \% ; 25.6 \%, 95 \%$ Cl: $25 \%-26.3 \%$; and $23.5 \%, 95 \% \mathrm{Cl}: 22.9 \%-24.1 \%)$ in the three waves, respectively. The prevalence rates were higher in the poorest wealth tertile than in the wealthiest tertile for the vast majority of diseases, except dyslipidaemia, diabetes or high blood sugar, cancer or malignant tumour, and liver diseases, which were either similar between the richest and poorest groups or higher among the wealthiest (Fig. 1). For chronic lung diseases, the prevalence was 1.4 times higher in the poorest than in the wealthiest tertile $(10.7 \%$ vs. $7.6 \%)$ in $2015-2016$; for arthritis or rheumatism, the prevalence was 1.37 times higher in the poorest than in the wealthiest tertile (32.9\% vs. $24.1 \%)$. For emotional, nervous, or psychiatric problems, the prevalence was 2.6 times higher (2.1\% vs. $0.8 \%)$ in the poorest than in the wealthiest tertile (Fig. 1 , Additional file 1: Table S4).

The estimated concentration indices showed that socioeconomic inequalities for dyslipidaemia, diabetes or high blood sugar, and cancer or malignant tumour were 0.1256 (95\% Cl: $0.1052-0.151), 0.098$ (0.0704-0.1244), and $0.1305(0.0528-0.215)$ in 2015-2016, respectively, which were indicative of prowealthy inequality (Table 2). The health inequalities for chronic lung diseases, heart disease, kidney disease, stomach or other digestive disease, psychiatric problems, memory-related disease, arthritis or rheumatism, and asthma tended to be greater among people with low SES (Fig. 2). To a large extent, relative inequalities in these diseases grew markedly over the study period. The concentration indices for hypertension and stroke were, respectively, $0.0151(-0.002-0.0307)$ and $0(-0.064-0.0519)$ in 20112012 and $-0.026(-0.04--0.012)$ and $-0.11(-0.16--0.065)$ in 2015-2016. These findings indicated a change in health equity from fair to unfair within a 5-year period (Table 2, Fig. 2). There was also significant pro-poor health inequality among comorbidities, with a concentration index $-0.056(-0.06-$ $-0.047)$ in 2015-2016.

Notably, the concentration index of the number of comorbidities among women $(-0.051,-0.05,-0.072)$ was more than twice that among men $(-0.023,-0.02,-0.032)$ in waves 1,2 , and 3 , respectively, and the gap was widening (Fig. 3, Additional file 1: Table S5). Additionally, regarding heart diseases, health equity was relatively fair among men, whereas health inequality was high among poor women. With pro-rich inequality, as seen in dyslipidaemia and diabetes or high blood sugar, the degree of inequality among women was smaller than that among men. 
Regarding comorbidities, urban residents had smaller health inequalities $(-0.035$ in $2011-2012,-0.036$ in 2013-2014, and -0.05 in 2015-2016); pro-poor health inequalities were larger among rural residents (-0.053 in 2011-2012, -0.064 in 2013-2014, and -0.08 in 2015-2016); and their confidence intervals did not overlap in 2015-2016. It is remarkable that compared with urban residents $(-0.038,-0.05$, $-0.057)$, pro-poor inequalities among rural residents $(-0.135,-0.17,-0.161)$ tripled with respect to heart disease in waves 1,2 , and 3 , respectively. In addition, the estimated concentration index of liver diseases in rural residents was $-0.097(-0.144--0.049)$ in 2015-2016, indicating pro-poor socioeconomic inequality in rural but not urban residents (Fig. 4, Additional file 1: Table S6).

Provinces ranking higher for GDP per capita also ranked higher in the degree to which health inequalities for dyslipidaemia, kidney disease, and asthma were pro-poor (Kendall's $\tau=-0.2328, p=0.015$; Kendall's $\tau$ $=-0.3545, p=0.0077 ;$ Kendall's $\tau=-0.2646, p=0.0079$, respectively; Fig. 5$)$. The variation in inequality among provinces explained by GDP per capita was 0.207 for dyslipidaemia, 0.2427 for kidney disease, and 0.2416 for asthma (Fig. 5, Additional file 1: Table S7).

\section{Discussion}

Our findings demonstrated obvious health disparities in several dimensions among middle-aged and elderly Chinese adults, calculated using the concentration index and corresponding $\mathrm{Cl}$ of 14 chronic diseases based on data from the CHARLS. The results indicated that absolute inequalities among women and rural residents were nearly twice those of men and urban residents in most instances. Conversely, for diabetes and dyslipidaemia, the prevalence was found to be more pro-rich among men and urban residents than among women and rural residents. Health inequalities for dyslipidaemia, kidney disease, and asthma were associated with provincial per capita GDP.

According to the general trends in socioeconomic inequalities in the 14 chronic diseases investigated, 9 showed negative concentration indices throughout the 6-year study, indicating that people with lower SES tended to have more chronic diseases in most cases. This is consistent with a prior study demonstrating considerable socioeconomic inequalities in the prevalence of chronic diseases [29]. A study in Germany revealed that low SES groups seem to face a double burden of increased levels of health impairments and lower levels of health-related quality of life once health is impaired [30]. SES is closely linked to the utilization of health services; wealthier communities may have better health services, resulting in a lower prevalence of chronic diseases.

With regard to diabetes, dyslipidaemia and cancer, people with higher SES are more likely to develop these diseases. A survey of the diabetic population in India showed that people with higher SES are more susceptible to diabetes than those with lower SES [31]. The prevalence of diabetes in economically developed regions is significantly higher than that in underdeveloped regions in China. Urbanization has led to changes in people's lifestyles, which has significantly reduced physical activity, accelerated the pace of life and placed people in long-term patterns of stress. All of these factors are involved in diabetes. Among Korean men, socioeconomic status was positively associated with dyslipidaemia. Interestingly, 
the risk of obesity also increased with income in Korean men. Families with high SES tend to enjoy a diet high in protein and fat and excessive alcohol consumption; these families are also less physically active. In addition, the most important factor in cancer control is early diagnosis. People with low SES have little access to adequate and high-quality medical care, which may result in fewer patients being detected.

The analysis of different groups according to sex and living environment revealed that health inequalities were mostly pro-poor but with different intensities across groups. For example, the inequality index of the number of comorbidities among women was twice as high as that among men. Sex-based social norms and constraints regarding economic autonomy lead to an unbalanced distribution of health resources towards men [32]. Compared with men, women are less independent and less physically active. Muchneeded health services for women have been neglected, ultimately leading to worse health conditions. If inequity is not addressed, health disparities will persist between men and women in China, with women remaining unable to receive adequate health services or achieve health equality with men.

With regard to living environment, our results indicated that pro-poor health inequalities among rural residents were larger than those among urban dwellers. It has been reported that urban-rural differences in health inequalities, influenced by SES, reflect the impact of the predominant dual urban-rural structure and household registration policy of China [33]. Furthermore, the health status and utilization of health services among elderly adults in urban areas are better than those among elderly adults in rural areas [5, 33]. Consistent with previous studies, differences in urban and rural health inequalities deepened during 2011-2016 [34,35]. The phenomenon of "poverty owing to illness" is widespread in rural areas of China. Qi and Wang found that the health gradient differs between urban and rural areas and that rural residents in China are more dependent on SES than urban residents are [36]. The imbalance between urban and rural areas in terms of economic development, public health expenditure, and health care has led to obvious urban-rural differences in the health status of residents. Thus, greater efforts are needed to reduce these health inequalities as well as the gap between residents of rural and urban areas. Such efforts include adopting policies targeting the poor, particularly the rural poor. Moreover, inequalities in comorbidities are high among middle-aged and older rural Chinese populations. The management of multimorbidity therefore warrants greater attention from health policy-makers, health care providers, and educators of health professionals in China as well as in other low- and middle-income countries [37].

We found that greater socioeconomic health inequalities exist in provinces with high GDP per capita, especially for dyslipidaemia, kidney disease, and asthma. On the one hand, the prevalence of asthma and kidney disease is pro-poor at the country level, with the problem more markedly pro-poor in provinces with higher GDP. This finding is consistent with a previous report that suboptimal asthma care and poor asthma control are common, even in developed areas of China [38]. Higher GDP per capita and higher health expenditure per capita do not seem to have led to lower rates of kidney disease and asthma. On the other hand, the prevalence of dyslipidaemia in China indicates pro-rich inequality; however, the opposite is true among those provinces with the highest GDP. This is similar to the situation in which lowincome people in developed countries have higher rates of dyslipidaemia and obesity [39]. The findings imply a major challenge to present and future case management for patients, particularly those in low- 
income groups who pay a large portion of health-care costs out-of-pocket. One potential policy response is to concentrate on raising the utilization of public health services by securing universal access without targeting any particular group. A comprehensive national medical service improvement programme is greatly needed.

With the increasing popularity of medical care data and the rapid development of big data analysis methods, the successful application of artificial intelligence (Al) in medical care has become possible. A recent review found emerging research showing the benefits of $A l$ in the management of cancer, heart diseases, stroke, vision impairment, dementia, and depression, which are common chronic diseases in China. At present, in the field of chronic diseases, Al can mainly assist doctors in diagnosis and relieve the shortage of medical resources. If we can have corresponding smart devices that can be used in daily life to test patients, manage patients' health status and provide feedback to doctors, patients will have a reduced need to go to a hospital for care and treatment. Therefore, Al may benefit patients of lower socioeconomic status with less access to medical care by doctors and enhance the cost-effectiveness of chronic disease management.

There are at least three limitations of this study. First, the CHARLS samples were not selected to be nationally representative of all age groups, and people younger than 45 years old were not included in the study; therefore, our results do not fully reflect China's overall health inequalities. Whereas the sampled populations were similar to the national population older than 45 years old with respect to major demographic and socioeconomic characteristics, estimates of health inequities among middle-aged and

elderly adults in China may be the most accurate. As mentioned earlier, a small percentage of our sample was excluded from the analysis owing to missing data. We did not impute missing wealth data, and these observations were excluded from our analyses, which assumes that missing wealth data are completely random in all counties. This assumption might bias our inequality estimates, although the direction of that bias is unclear. Second, the data used here were based on self-reported chronic conditions and are therefore subject to potential recall bias. However, we applied a recall correction for responses given during follow-up interviews, which could remediate the potential biases; in addition, the available data from other studies of chronic diseases provide some support for the accuracy of selfreported data. Third, quantitative analyses revealed but could not provide a causal explanation for socioeconomic inequalities. The next step requires multidisciplinary research (e.g., a decomposition of the concentration index) to clarify the observed variations.

\section{Conclusion}

Our study showed significant health inequalities in 2011-2016 among middle-aged and older adults in China in several dimensions. These results imply that enhanced awareness is urgently needed to reduce inequalities. Further studies are needed, as well as interventions to reduce health inequalities in China.

\section{Abbreviations}


SD: standard deviation; Cl: confidence interval; SES: socioeconomic status

\section{Declarations}

\section{Acknowledgment}

We would like to acknowledge the CHARLS team for the publicly available data.

\section{Authors' contributors}

HPS designed the study; XQQ and JFZ managed statistical analysis and interpretation of the results; $\mathrm{XQQ}, \mathrm{HPS}, \mathrm{LYH}$ and ZC wrote the manuscript; all authors reviewed the manuscript.

\section{Funding}

This study was funded by Jiangsu Key Laboratory of Preventive and Translational Medicine for Geriatric Diseases (KJS1513), National Natural Science Foundation of China (81402761), the Foundation Research Project of Jiangsu Province (BK20140361), Suzhou Xiangcheng district people's livelihood science and technology project (XJ201655, XJ201706).

\section{Availability of data and materials}

The datasets used and/or analysed during the current study are available from the corresponding author on reasonable request

\section{Ethics approval and consent to participate}

All examinations were conducted after obtaining informed consent from participants. The Biomedical Ethics Committee of Peking University approved the survey (The approval number is IRB0000105211015), and the conduct of the study adhered to the principles of the Declaration of Helsinki.

\section{Consent for publication}

Not applicable

\section{Competing interests}

We declare no competing interests.

\section{References}

1. Wang Y, Wang J, Maitland E, Zhao YH, Nicholas S, Lu MS: Growing old before growing rich: inequality in health service utilization among the mid-aged and elderly in Gansu and Zhejiang Provinces, China. Bmc Health Services Research 2012, 12. 
2. Adeloye D, Chan KY, Rudan I, Campbell H: An estimate of asthma prevalence in Africa: a systematic analysis. Croat Med J 2013, 54:519-531.

3. Li C, Sun L, Wang Y: The Impact of Chronic Disease on Healthy Life Expectancy of the Elderly in China. Chinese Journal of Health Statistics 2017.

4. Cai J, Coyte PC, Zhao H: Decomposing the causes of socioeconomic-related health inequality among urban and rural populations in China: a new decomposition approach. International Journal for Equity in Health 2017, 16:128.

5. Zhong Y, Gao Y, Wang J: Study on the Equity in the Elderly with Different Socioeconomic Status. Chinese Health Economics 2013:21.

6. Li H, Hanewald K, Wu S: Estimating Healthy Life Expectancy: A Province-by-Province Study for China. Social Science Electronic Publishing 2017.

7. Li H, Lv L, Wang X: Research on the Health Fairness of Residents in Different Economic Status in Weihai City. Chinese Health Service Management 2008, 25:776.

8. World Health O: World health statistics 2016. Monitoring health for the SDGs Sustainable Development Goals. Geneva Switzerland Who 2016, 41:293-328.

9. Dingwan C, He F: The Research on the Health Equality in Different Social Stratification. Chinese Health Economics 2006, 25:88.

10. Kagamimori S, Gaina A, Nasermoaddeli A: Socioeconomic status and health in the Japanese population. Soc Sci Med 2009, 68:2152.

11. Bendavid E: Changes in child mortality over time across the wealth gradient in less-developed countries. Pediatrics 2014, 134:e1551-1559.

12. Victora CG, Barros AJ, Fran?a GV, da Silva IC, Carvajal-Velez L, Amouzou A: The contribution of poor and rural populations to national trends in reproductive, maternal, newborn, and child health coverage: analyses of cross-sectional surveys from 64 countries. The Lancet Global health 2017, 5:e402-e407.

13. Alkenbrack S, Chaitkin M, Zeng W, Couture T, Sharma S: Did Equity of Reproductive and Maternal Health Service Coverage Increase during the MDG Era? An Analysis of Trends and Determinants across 74 Low- and Middle-Income Countries. PloS one 2015, 10:e0134905.

14. Victora CG, Barros AJ, Axelson H, Bhutta ZA, Chopra M, Fran?a GV, Kerber K, Kirkwood BR, Newby H, Ronsmans C, Boerma JT: How changes in coverage affect equity in maternal and child health interventions in 35 Countdown to 2015 countries: an analysis of national surveys. Lancet 2012, 380:1149-1156.

15. Ross J: Improved Reproductive Health Equity Between the Poor and the Rich: An Analysis of Trends in 46 Low- and Middle-Income Countries. Global health, science and practice 2015, 3:419-445.

16. Restrepo-Méndez MC, Barros AJ, Wong KL, Johnson HL, Pariyo G, Fran?a GV, Wehrmeister FC, Victora CG: Inequalities in full immunization coverage: trends in low- and middle-income countries. Bulletin of the World Health Organization 2016, 94:794-805B. 
17. Yaohui Z: China Health and Retirement Longitudinal Study[2011 baselinel.

18. Yaohui Z: China Health and Retirement Longitudinal Study $\square 2013$ wave2".

19. Zhao Y, Hu Y, Smith JP, Strauss J, Yang G: Cohort profile: the China Health and Retirement Longitudinal Study (CHARLS). Int J Epidemiol 2014, 43:61.

20. Filmer D, Pritchett LH: Estimating wealth effects without expenditure data-or tears: an application to educational enrollments in states of India. Demography 2001, 38:115.

21. Vyas S, Kumaranayake L: Constructing socio-economic status indices: How to use principal components analysis. Health Policy and Planning 2006, 21:459.

22. Analyzing Health Equity Using Household Survey Data.

23. Wang Z, Li X, Chen M: Catastrophic health expenditures and its inequality in elderly households with chronic disease patients in China. International Journal for Equity in Health,14,1(2015-01-20) 2015, 14:8.

24. Analyzing health equity using household survey data: a guide to techniques and their implementation. The World Bank; 2008.

25. Wagstaff $A$ : The bounds of the concentration index when the variable of interest is binary, with an application to immunization inequality. Health Econ 2005, 14:429.

26. Wagstaff A: The concentration index of a binary outcome revisited. Health Econ 2011, 20:1155.

27. Jenkins R: Income-related inequality in mental health in Britain : the concentration index approach. 2007:1037.

28. Hajizadeh M, Campbell MK, Sarma S: Socioeconomic inequalities in adult obesity risk in Canada: trends and decomposition analyses. European Journal of Health Economics Hepac Health Economics in Prevention \& Care 2013, 15:203.

29. Efron B, Tibshirani R: Bootstrap Methods for Standard Errors, Confidence Intervals, and Other Measures of Statistical Accuracy. Statistical Science 1986, 1:54.

30. Vukovi D, Bjegovi V, Vukovi G: Prevalence of chronic diseases according to socioeconomic status measured by wealth index: health survey in Serbia. Croatian Medical Journal 2008, 49:832.

31. Mielck A, Vogelmann M, Leidl R: Health-related quality of life and socioeconomic status: inequalities among adults with a chronic disease. Health and Quality of Life Outcomes 2014, 12:58.

32. Yan-ling MAO: Gender analysis of health equity. HEALTH ECONOMICS RESEARCH 2010:15.

33. Zhao R: The Urban-Rural Differences of the Influence of Socioeconomic Status on Health Conditions. Future and Development 2015:21.

34. Zimmer Z, Kaneda T, Spess L: An Examination of Urban Versus Rural Mortality in China Using Community and Individual Data. J Gerontol B Psychol Sci Soc Sci 2007, 62:S349.

35. Liang J, McCarthy JF, Jain A, Krause N, Bennett JM, Gu S: Socioeconomic gradient in old age mortality in Wuhan, China. Journals of Gerontology - Series B Psychological Sciences and Social Sciences 2000 . 
36. Liangshu Q, Chengwei W: Health Gradient and Rural-urban Disparity:An Empirical Study Based on Data from 9 Provinces of China. Chinese Health Economics 2011, 30:11.

37. Chen $\mathrm{H}$, Cheng $\mathrm{M}$, Zhuang $\mathrm{Y}$, Broad JB: Multimorbidity among middle-aged and older persons in urban China: Prevalence, characteristics and health service utilization. Geriatrics \& gerontology international 2018, 18:1447-1452.

38. Ibrahim WH, Suleiman NN, El-Allus F, Suleiman J, Elbuzidi AA, Guerrero MD, Guerrero AN, Akkari A, Karim H, Awouda SA, Suliman S: The burden of adult asthma in a high GDP per capita country: the QASMA study. Annals of allergy, asthma \& immunology : official publication of the American College of Allergy, Asthma, \& Immunology 2015, 114:12-17.

39. Ezzati M, Vander Hoorn S, Lawes CM, Leach R, James WP, Lopez AD, Rodgers A, Murray CJ: Rethinking the "diseases of affluence" paradigm: global patterns of nutritional risks in relation to economic development. PLoS medicine 2005, 2:e133.

\section{Tables}

Table 1 Characteristics of survey respondents in China: 2011-2012, 2013-2014, 2015-2016

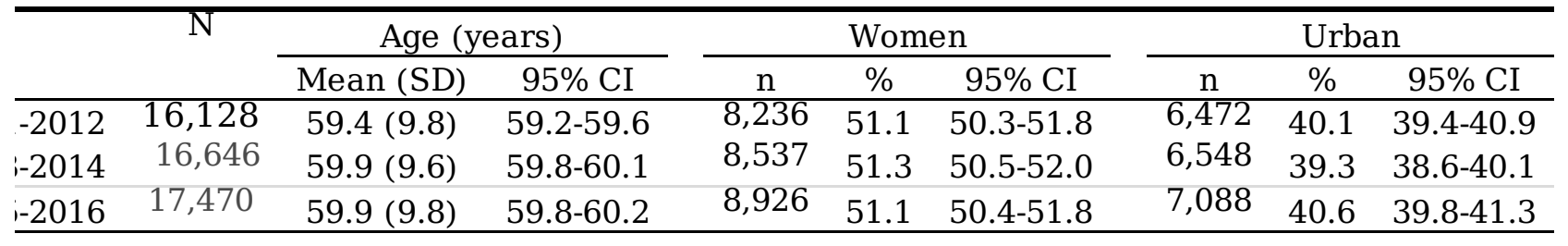

Abbreviation: SD, standard deviation; CI, confidence interval.

Table 2 Concentration indices of 14 chronic diseases and comorbidities: 2011-2012, 2013-2014, 2015-2016 


\begin{tabular}{|c|c|c|c|}
\hline Disease & $\begin{array}{c}2011-2012 \\
\text { CI }(95 \% \mathrm{CI})\end{array}$ & $\begin{array}{c}2013-2013 \\
\text { CI(95\%CI) }\end{array}$ & $\begin{array}{c}2015-2016 \\
\text { CI(95\%CI) }\end{array}$ \\
\hline Hypertension & $\begin{array}{l}0.0151 \\
(-0.002,0.0307)\end{array}$ & $\begin{array}{l}-0.008 \\
(-0.022,0.0059)\end{array}$ & $\begin{array}{l}-0.026 \\
(-0.04,-0.012)\end{array}$ \\
\hline Dyslipidemia & $\begin{array}{l}0.1874 \\
(0.1594,0.21)\end{array}$ & $\begin{array}{l}0.1714 \\
(0.1486,0.19)\end{array}$ & $\begin{array}{l}0.1256 \\
(0.1052,0.151)\end{array}$ \\
\hline Diabetes or high blood sugar & $\begin{array}{l}0.1362 \\
(0.102,0.1753)\end{array}$ & $\begin{array}{l}0.1303 \\
(0.099,0.1598)\end{array}$ & $\begin{array}{l}0.098 \\
(0.0704,0.1244)\end{array}$ \\
\hline Cancer or malignant tumor & $\begin{array}{l}0.1163 \\
(0.0228,0.2089)\end{array}$ & $\begin{array}{l}0.0907 \\
(0.0113,0.178)\end{array}$ & $\begin{array}{l}0.1305 \\
(0.0528,0.215)\end{array}$ \\
\hline Chronic lung diseases & $\begin{array}{l}-0.143 \\
(-0.167,-0.116)\end{array}$ & $\begin{array}{l}-0.123 \\
(-0.146,-0.102)\end{array}$ & $-0.15(-0.18,-0.13)$ \\
\hline Liver disease & $\begin{array}{l}-0.044 \\
(-0.089,0.001)\end{array}$ & $\begin{array}{l}0.009 \\
(-0.031,0.0532)\end{array}$ & $\begin{array}{l}-0.017 \\
(-0.059,0.0206)\end{array}$ \\
\hline Heart disease & $-0.03(-0.057,-0.007)$ & $\begin{array}{l}-0.059 \\
(-0.081,-0.037)\end{array}$ & $\begin{array}{l}-0.066 \\
(-0.088,-0.043)\end{array}$ \\
\hline Stroke & $0(-0.064,0.0519)$ & $\begin{array}{l}-0.014 \\
(-0.067,0.0341)\end{array}$ & $-0.11(-0.16,-0.065)$ \\
\hline Kidney disease & $\begin{array}{l}-0.075 \\
(-0.106,-0.041)\end{array}$ & $\begin{array}{l}-0.062 \\
(-0.093,-0.029)\end{array}$ & $\begin{array}{l}-0.084 \\
(-0.116,-0.04)\end{array}$ \\
\hline Stomach or other digestive disease & $-0.063(-0.08,-0.047)$ & $\begin{array}{l}-0.067 \\
(-0.081,-0.052)\end{array}$ & $\begin{array}{l}-0.071 \\
(-0.085,-0.056)\end{array}$ \\
\hline $\begin{array}{l}\text { Emotional, nervous, or psychiatric } \\
\text { problems }\end{array}$ & $\begin{array}{l}-0.188 \\
(-0.258,-0.115)\end{array}$ & $\begin{array}{l}-0.094 \\
(-0.181,-0.013)\end{array}$ & $\begin{array}{l}-0.232 \\
(-0.302,-0.16)\end{array}$ \\
\hline Memory_related disease & $\begin{array}{l}-0.089 \\
(-0.162,-0.016)\end{array}$ & $-0.1(-0.175,-0.056)$ & $\begin{array}{l}-0.151 \\
(-0.207,-0.088)\end{array}$ \\
\hline Arthritis or rheumatism & $-0.101(-0.11,-0.089)$ & $\begin{array}{l}-0.101 \\
(-0.112,-0.09)\end{array}$ & $\begin{array}{l}-0.122 \\
(-0.133,-0.11)\end{array}$ \\
\hline Asthma & $-0.16(-0.206,-0.115)$ & $\begin{array}{l}-0.162 \\
(-0.202,-0.12)\end{array}$ & $-0.2(-0.24,-0.162)$ \\
\hline Count of comorbidity & $\begin{array}{l}-0.037 \\
(-0.046,-0.029)\end{array}$ & $-0.038(-0.04,-0.02)$ & $\begin{array}{l}-0.056 \\
(-0.06,-0.047) \\
\end{array}$ \\
\hline
\end{tabular}

Abbreviation: CI, confidence interval.

Figures 


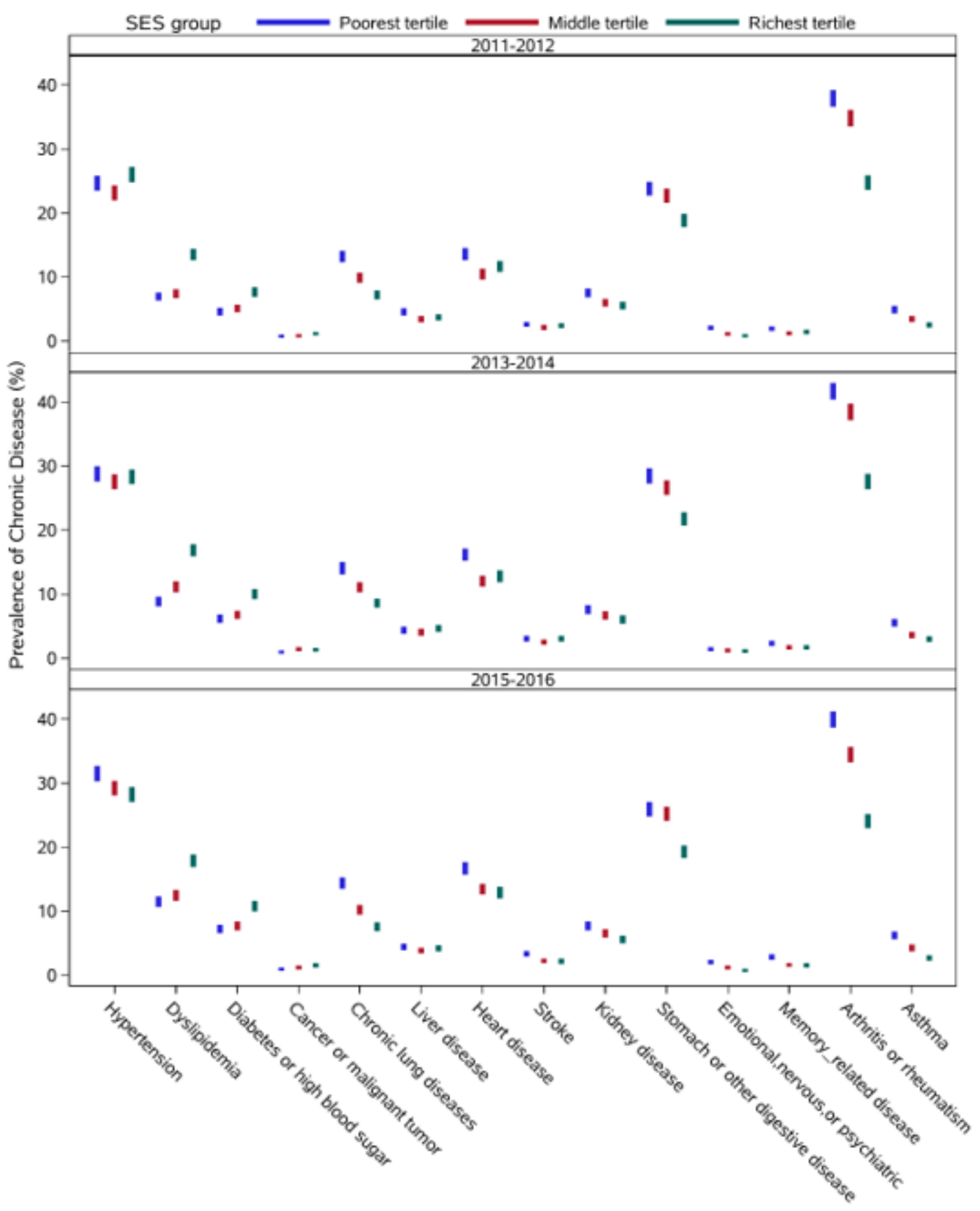

Figure 2

High-low plot showing $95 \%$ confidence intervals for the prevalence of 14 chronic diseases, by tertiles according to socioeconomic status (SES) 


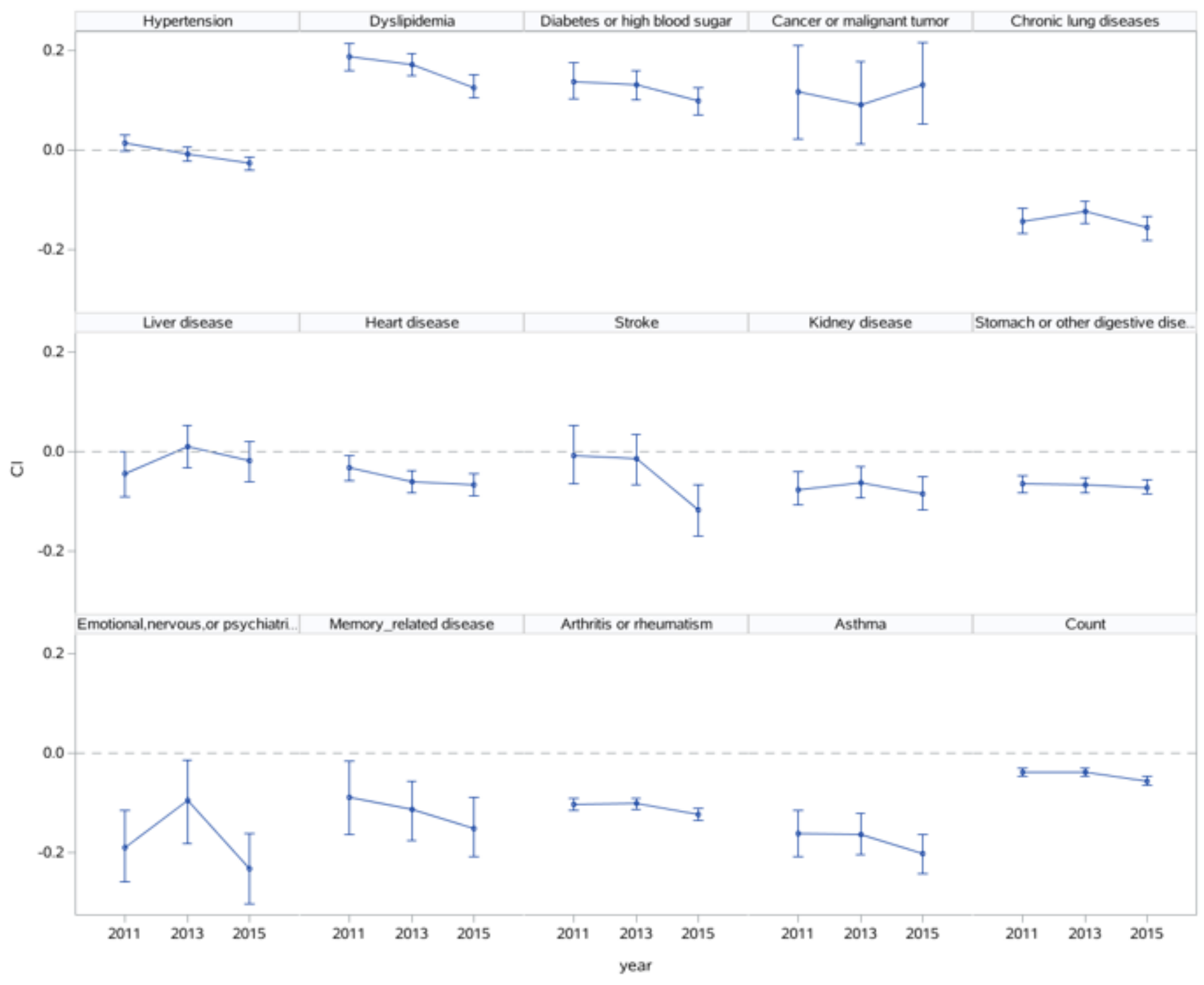

Figure 4

Trends of concentration indices for 14 chronic diseases and comorbidities, 2011-2016 


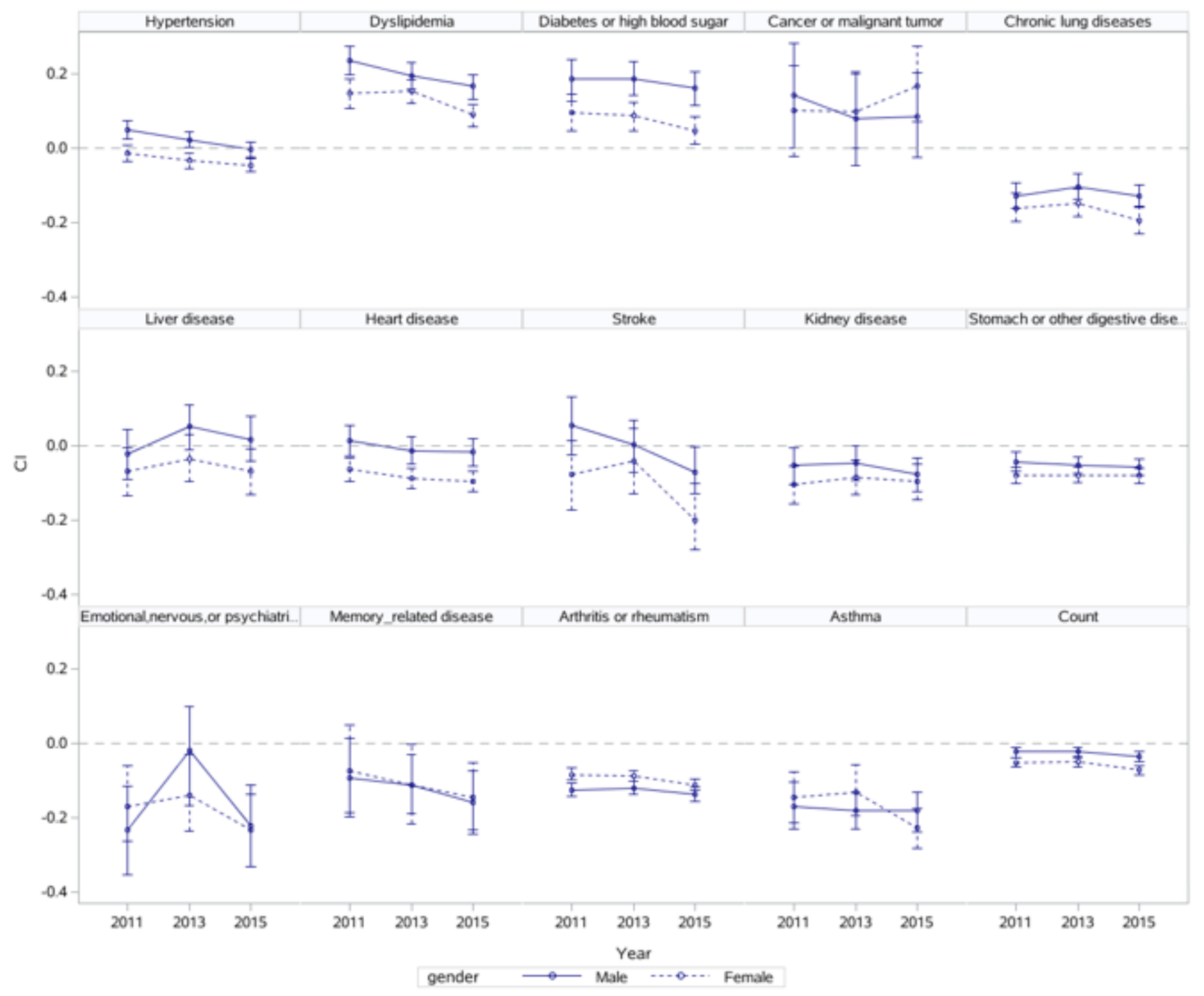

Figure 6

Trends of concentration indices for 14 chronic diseases and comorbidities by subpopulations according to sex, 2011-2016 


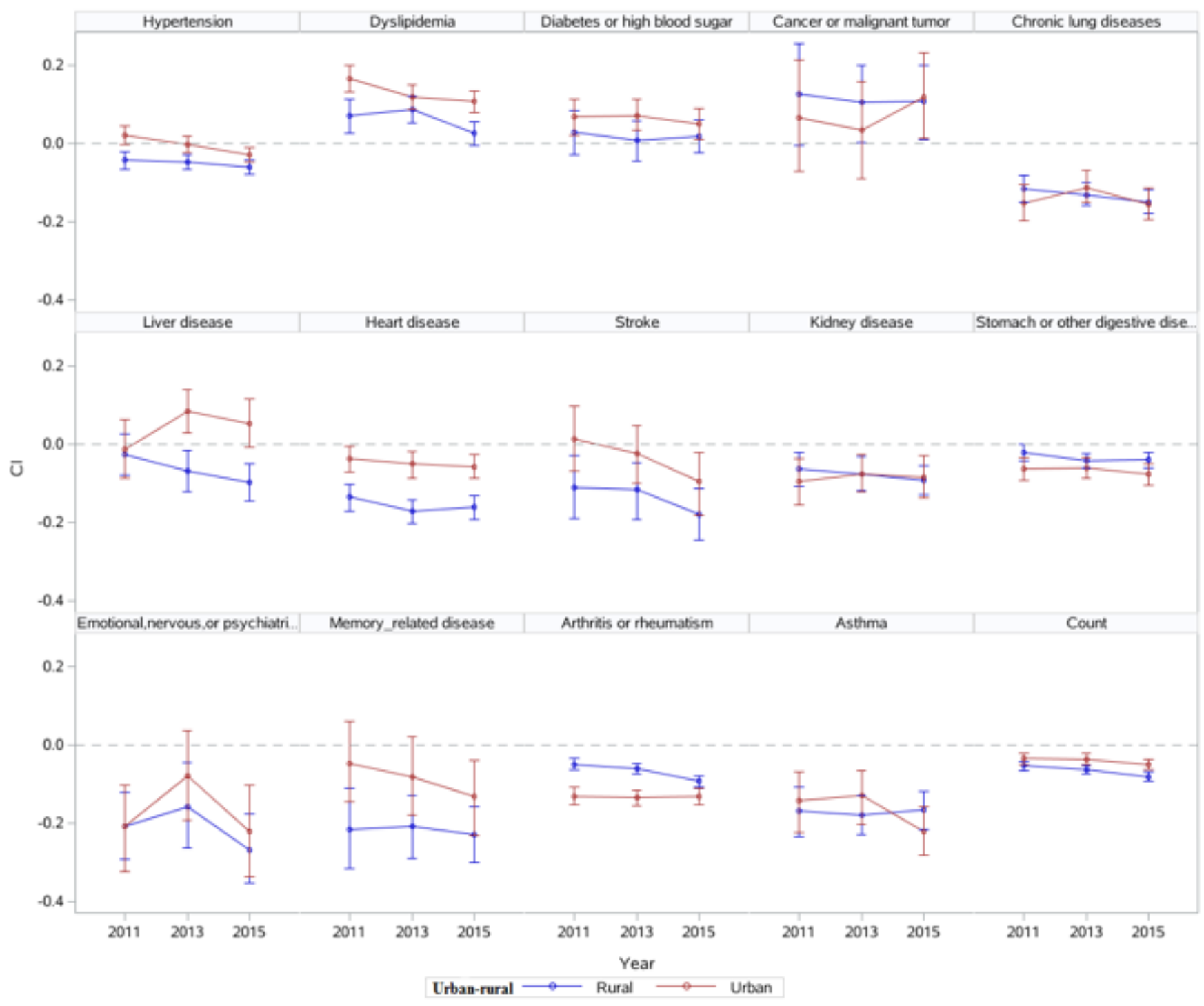

Figure 8

Trends of concentration indices for 14 chronic diseases and comorbidities by subpopulations according to rural or urban residence, 2011-2016 


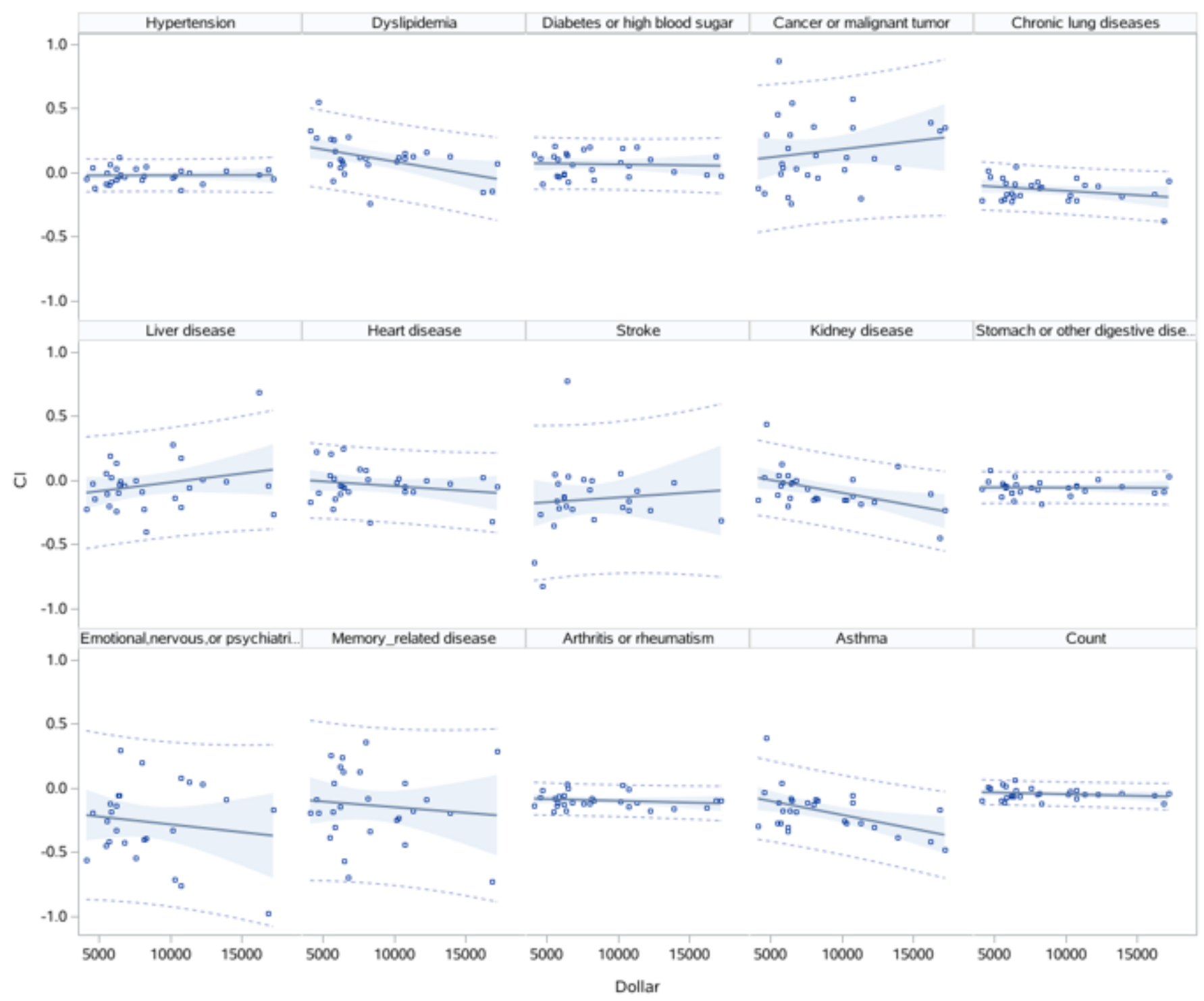

Figure 10

Scatter plots of concentration indices for inequality in 14 chronic diseases and comorbidities against GDP per capita, 2015-2016

\section{Supplementary Files}

This is a list of supplementary files associated with this preprint. Click to download.

- Equation1.jpg

- Equation2.jpg

- Additionalfile1.docx

- Additionalfile1.docx

- Equation2.jpg 
- Equation1.jpg

Page 20/20 